\title{
Impaired hepatic lipid synthesis from polyunsaturated fatty acids in TM6SF2 E167K variant carriers with NAFLD
}

\section{Luukkonen, Panu K.}

2017-07

Luukkonen , P K , Zhou , Y, Haridas , N P A , Dwivedi , O P , Hyotylainen , T , Ali , A , Juuti , A, Leivonen, M , Tukiainen , T , Ahonen, L , Scott , E , Palmer , J M , Arola , J , Orho-Melander , M , Vikman , P , Anstee , Q M , Olkkonen , V M , Oresic , M , Groop , L \& Yki-Jarvinen , H 2017 , ' Impaired hepatic lipid synthesis from polyunsaturated fatty acids in TM6SF2 E167K variant carriers with NAFLD ', Journal of Hepatology , vol. 67 , no. 1 , pp. 128-136 . https://doi.org/10.1016/j.jhep.2017.02.014

http://hdl.handle.net/10138/236998

https://doi.org/10.1016/j.jhep.2017.02.014

unspecified

publishedVersion

Downloaded from Helda, University of Helsinki institutional repository.

This is an electronic reprint of the original article.

This reprint may differ from the original in pagination and typographic detail.

Please cite the original version. 


\title{
Impaired hepatic lipid synthesis from polyunsaturated fatty acids in TM6SF2 E167K variant carriers with NAFLD
}

\author{
Panu K. Luukkonen ${ }^{1,2, *}$, You Zhou ${ }^{2,3}$, Nidhina Haridas P.A. ${ }^{2}$, Om P. Dwivedi ${ }^{4}$, Tuulia Hyötyläinen ${ }^{5}$, \\ Ashfaq Ali ${ }^{6}$, Anne Juuti ${ }^{7}$, Marja Leivonen ${ }^{7}$, Taru Tukiainen ${ }^{4}$, Linda Ahonen ${ }^{6}$, Emma Scott $^{8}$, \\ Jeremy M. Palmer ${ }^{8}$, Johanna Arola ${ }^{9}$, Marju Orho-Melander ${ }^{10}$, Petter Vikman ${ }^{10}$, Quentin M. Anstee ${ }^{8}$, \\ Vesa M. Olkkonen ${ }^{2}$, Matej Orešič ${ }^{11}$, Leif Groop ${ }^{4,10}$, Hannele Yki-Järvinen ${ }^{1,2}$ \\ ${ }^{1}$ Department of Medicine, University of Helsinki and Helsinki University Hospital, Helsinki, Finland; ${ }^{2}$ Minerva Foundation Institute for Medical \\ Research, Helsinki, Finland ${ }^{3}$ Systems Immunity University Research Institute and Division of Infection and Immunity, School of Medicine, Cardiff \\ University, Cardiff, United Kingdom, ${ }^{4}$ Institute for Molecular Medicine Finland, Helsinki, Finland; ${ }^{5}$ Department of Chemistry, Örebro University, \\ Örebro, Sweden; ${ }^{6}$ Steno Diabetes Center, Gentofte, Denmark; ${ }^{7}$ Department of Surgery, University of Helsinki and Helsinki University Hospital, \\ Helsinki, Finland; ${ }^{8}$ Institute of Cellular Medicine, Faculty of Medical Sciences, Newcastle University, Newcastle upon Tyne, United Kingdom; \\ ${ }^{9}$ Department of Pathology, University of Helsinki and Helsinki University Hospital, Helsinki, Finland; ${ }^{10}$ Lund University, Malmö, Sweden; \\ ${ }^{11}$ Turku Centre for Biotechnology, University of Turku and Åbo Akademi University, Turku, Finland
}

\begin{abstract}
Background: Carriers of the transmembrane 6 superfamily member 2 E167K gene variant (TM6SF2 ${ }^{\mathrm{EK} / \mathrm{KK}}$ ) have decreased expression of the TM6SF2 gene and increased risk of NAFLD and NASH. Unlike common 'obese/metabolic' NAFLD, these subjects lack hypertriglyceridemia and have lower risk of cardiovascular disease. In animals, phosphatidylcholine (PC) deficiency results in a similar phenotype. PCs surround the core of VLDL consisting of triglycerides (TGs) and cholesteryl-esters (CEs). We determined the effect of the TM6SF2 E167K on these lipids in the human liver and serum and on hepatic gene expression and studied the effect of TM6SF2 knockdown on hepatocyte handling of these lipids.
\end{abstract}

Methods: Liver biopsies were taken from subjects characterized with respect to the TM6SF2 genotype, serum and liver lipidome, gene expression and histology. In vitro, after TM6SF2 knockdown in HuH-7 cells, we compared incorporation of different fatty acids into TGs, CEs, and PCs.

Results: The TM6SF2 ${ }^{\mathrm{EK} / \mathrm{KK}}$ and TM6SF2 ${ }^{\mathrm{EE}}$ groups had similar age, gender, BMI and HOMA-IR. Liver TGs and CEs were higher and liver PCs lower in the TM6SF2 ${ }^{\mathrm{EK} / \mathrm{KK}}$ than the TM6SF2 ${ }^{\mathrm{EE}}$ group $(p<0.05)$. Polyunsaturated fatty acids (PUFA) were deficient in liver and serum TGs and liver PCs but hepatic free fatty acids were relatively enriched in PUFA $(p<0.05)$. Incorporation of PUFA into TGs and PCs in TM6SF2 knockdown hepatocytes was decreased $(p<0.05)$. Hepatic expression of TM6SF2 was

\footnotetext{
Keywords: Transmembrane 6 superfamily member 2; Triglycerides; Cholestero esters; Arachidonic acid; Phosphatidylcholines; Non-alcoholic fatty liver disease: Lipogenesis; Genotype; Hepatocytes; Fatty acids.

Received 28 November 2016; received in revised form 7 February 2017; accepted 10 February 2017; available online 22 February 2017

* Corresponding author. Address: University of Helsinki and Helsinki University Hospital, Department of Medicine, Tukholmankatu 8, 00290 Helsinki, Finland. Tel.: +358 29412 5708; fax: +35894717 1896 .

E-mail address: panu.luukkonen@fimnet.fi (P.K. Luukkonen).
}

decreased in variant carriers, and was co-expressed with genes regulated by PUFAs.

Conclusions: Hepatic lipid synthesis from PUFAs is impaired and could contribute to deficiency in PCs and increased intrahepatic TG in TM6SF2 E167K variant carriers.

(C) 2017 European Association for the Study of the Liver. Published by Elsevier B.V. All rights reserved.

\section{Introduction}

Common non-alcoholic fatty liver disease (NAFLD) is associated with features of the metabolic syndrome, such as hypertriglyceridemia, and increased risk of cardiovascular disease (CVD). ${ }^{1}$ In 2014, a non-synonymous adenine-to-guanine substitution (rs58542926) replacing glutamate at residue 167 with lysine (E167K) in the transmembrane 6 superfamily member 2 (TM6SF2) protein was shown to increase liver fat content ${ }^{2,3}$ and the risk of liver fibrosis. ${ }^{4}$ In these and several subsequent studies, variant allele carriers (TM6SF2 ${ }^{\mathrm{EK} / \mathrm{KK}}$ ) were found to be neither more obese nor more insulin-resistant than non-carriers $\left(T M 6 S F 2^{\mathrm{EE}}\right) .^{2,5-12}$ In contrast to most cases with 'obese/metabolic' NAFLD, TM6SF2 variant allele carriers have normal ${ }^{5,6,10,11}$ or decreased $^{2,8-10,12}$ plasma TG concentrations and a reduced risk of CVD. ${ }^{12,13}$

The exact function of the TM6SF2 variant is unknown. In hepatocytes, TM6SF2 minor allele carriage is associated with decreased TM6SF2 protein expression. ${ }^{2}$ TM6SF2 siRNA inhibition in hepatocytes increases cellular TG concentrations and reduces TG secretion, while overexpression of TM6SF2 has the opposite effect. $^{3}$ In mice, hepatic knockdown of TM6SF2 increases liver triglycerides (TG) and cholesteryl esters (CE), the main constituents of the hydrophobic core of very low-density lipoprotein (VLDL) particles. ${ }^{2}$ 
Hepatic steatosis, reduced hepatic TG secretion, and protection from CVD characterize animals with deletion of genes that are involved in phosphatidylcholine (PC) synthesis such as CTP: phosphocholine cytidylyltransferase alpha $(C T \alpha)$ and phosphatidylethanolamine N-methyltransferase (PEMT). ${ }^{14,15}$ A similar phenotype also results from deletion of lysophosphatidylcholine acyltransferase 3 (LPCAT3), which decreases hepatic concentrations of polyunsaturated PCs. ${ }^{16}$ PCs are the only phospholipids required for assembly of VLDL particles. ${ }^{15}$ PC deficiency increases intrahepatic degradation of VLDL particles and thereby reduces their secretion. ${ }^{15}$ Dietary restriction of choline, a precursor of PC, increases liver fat in humans. ${ }^{17}$ Consumption of a methionine-choline-deficient diet, a widely used in vivo experimental model for NAFLD and NASH, reduces hepatic PC synthesis and plasma TGs and increases liver TGs without inducing insulin resistance. $^{15,18}$ In humans, a polymorphism in PEMT, which reduces $\mathrm{PC}$ synthesis, may be more frequent in participants with NAFLD ${ }^{19-21}$ and in participants with non-alcoholic steatohepatitis (NASH) ${ }^{22}$ than in those without, and characterizes participants who develop NAFLD on a choline-depleted diet. ${ }^{23}$ Since these changes resemble those of carriers of the TM6SF2 E167K gene variant, ${ }^{2-13}$ we hypothesized that reduced PC synthesis might characterize carriers of the E167K variant.

In the present study, we profiled hepatic gene expression and measured concentrations of TGs, CEs, PCs and free fatty acids (FFA) in human liver biopsy samples from carriers (TM6SF2 ${ }^{\mathrm{EK} / \mathrm{KK}}$ ) and non-carriers $\left(T M 6 S F 2^{\mathrm{EE}}\right.$ ) of the TM6SF2 E167K variant. Since the in vivo results seemed to support our hypothesis, we directly measured incorporation of saturated (16:0, palmitic acid), monounsaturated (18:1, oleic acid), and polyunsaturated fatty acids (20:4, arachidonic acid) into TG, CE, and PC pools in TM6SF2 knockdown HuH-7 cells. In addition, we measured circulating concentrations and composition of TGs, CEs and PCs in carriers and non-carriers of the gene variant.

\section{Materials and methods}

Study participants

Ninety participants were recruited from a cohort of patients undergoing laparoscopic bariatric surgery. Following a phone interview and a separate clinical study visit (vide infra), participants were eligible if they met the following inclusion criteria:

a. Age 18 to 75 years.

b. No known acute or chronic disease except for features of the metabolic syndrome based on medical history, physical examination and standard laboratory tests (complete blood count, serum creatinine, electrolyte concentrations)

c. Non-diabetic based on $2 \mathrm{~h}$ plasma glucose concentrations ( $\leqslant 11 \mathrm{mmol} / \mathrm{l})$ during a $75 \mathrm{~g}$ oral glucose tolerance test.

d. Alcohol consumption less than $20 \mathrm{~g}$ per day in women and less than $30 \mathrm{~g}$ per day in men.

e. No clinical or biochemical evidence of other liver disease, or clinical signs or symptoms of inborn errors of metabolism.

f. No use of drugs or toxins influencing liver steatosis.

Elevated liver enzymes (alanine aminotransferase (ALT) and aspartate aminotransferase (AST)) were not exclusion criteria. Participants with diabetes were excluded from the present study, as we wished to study the impact of the TM6SF2 genotype at rs58542926 rather than that of insulin resistance/diabetes and antidiabetic drugs on the human liver lipidome. The effect of the latter was reported earlier in a larger $(n=125)$ group of participants, which included patients with diabetes. ${ }^{24}$ The ethics committee of the Hospital District of Helsinki and Uusimaa

\section{JOURNAL OF HEPATOLOGY}

approved the study, which was conducted in accordance with the Declaration of Helsinki. Each participant provided written informed consent after the nature and potential risks of the study had been fully explained.

Metabolic study

One week prior to surgery, all participants were invited to a clinical visit for metabolic characterization after an overnight fast. Anthropometric measurements (body weight, height and waist circumference) were taken, an intravenous cannula inserted in an antecubital vein and blood collected for measurement of $\mathrm{HbA}_{1}$ serum insulin and adiponectin, plasma glucose, LDL- and HDL-cholesterol, triglyceride, AST, ALT, ALP, GGT and albumin concentrations and for genotyping of PNPLA3 rs738409 and TM6SF2 rs58542926 as previously described. ${ }^{24}$

The participants underwent an oral glucose $(75 \mathrm{~g})$ tolerance test (OGTT). ${ }^{25}$ HOMA-IR ${ }^{26}$ and Matsuda insulin sensitivity index ${ }^{27}$ were used as surrogates for insulin resistance. Body weight of the participants did not differ between the time of the metabolic study and surgery $(128.9 \pm 2.3$ and $128.5 \pm 2.3 \mathrm{~kg}$; nonsignificant [NS]). The participants were divided into groups based on their TM6SF2 rs58542926 genotype (E167K variant allele carriers, TM6SF2 ${ }^{\mathrm{EK} / \mathrm{KK}}, \mathrm{n}=10$ and noncarriers, $T M 6 S F 2^{\mathrm{EE}}, \mathrm{n}=80$ ).

Liver biopsies and liver histology

Routine wedge biopsies of the liver were taken immediately at the beginning of the surgery. Part of the biopsy was snap-frozen in liquid nitrogen for subsequent analysis of molecular lipids and gene expression; the other part was sent to an experienced liver pathologist (J.A.) for blinded analysis of liver histology according to the criteria proposed by Brunt et al. ${ }^{28}$ Liver fat was quantified as the percentage of hepatocytes with macrovesicular steatosis.

Hepatic lipidomic and free fatty acid analysis

Hepatic lipidomic analyses of TGs, CEs and PCs were performed using a Q-TOF Premier (Waters, Milford, MA) quadrupole time-of-flight mass spectrometer combined with an Acquity Ultra Performance liquid chromatograph (LC) (Waters, Milford, MA). Liquid chromatography methods are described in the Supplementary material. Hepatic FFAs were analysed using an Agilent 6890 gas chromatograph equipped with a split/splitless injector (Agilent Technologies, Santa Clara, CA), cryogenic dual-stage modulator and time-of-flight mass spectrometer (Leco Corp., St. Joseph, MI, USA) as described in detail in Supplementary material. All analyses were blinded.

Hepatic RNA sequencing

Since the results (vide infra) suggested that TM6SF2 might be involved in synthesis of polyunsaturated lipids, we examined hepatic gene expression using RNA sequencing. For this, liver samples were available from 69 of the original cohort. An additional 25 participants were recruited using the same criteria as the original cohort. The characteristics of these participants $(n=94)$ are shown in Table S2. The samples were prepared using Illumina TruSeq RNA Sample Preparation Kit (Illumina, San Diego, CA) and sequencing was performed using a pairedend 101 base pair protocol on HiSeq2000 platform (Illumina, San Diego, CA). The methods for RNA sequencing, data processing and pathway analysis are described in detail in the Supplementary material.

Serum lipidomic analysis

Serum lipidomic analyses of TGs, CEs and PCs were analysed using an ultra-highperformance liquid chromatography quadrupole time-of-flight mass spectrometry (UHPLC-Q-TOF-MS). The UHPLC was a 1290 Infinity system (Agilent Technologies, Santa Clara, CA) and the mass spectrometer a 6550 iFunnel Q-TOF (Agilent Technologies, Santa Clara, CA). Details of the sample preparations and data preprocessing steps are given in Supplementary material.

\section{In vitro experiments}

Stably transduced and transiently silenced $\mathrm{HuH}-7$ human hepatoma derived cells were used as in vitro hepatocyte models. The former was established by transducing HuH-7 cells with lentiviral particles expressing shRNA against TM6SF2 


\section{Research Article}

(TRCN0000254085, Sigma-Aldrich, St. Louis, MO) and a control non-targeting shRNA (SHC002V, Sigma-Aldrich), and the latter by using siRNA against TM6SF2 (SI04187946, Qiagen, Hilden, Germany) and a negative control (SI03650318, Qiagen) in HuH-7 cells. Silencing efficiency by quantitative polymerase chain reaction, lipid droplet quantification, and incorporation of tritiated fatty acids $\left(\left[9,10-{ }^{3} \mathrm{H}(\mathrm{N})\right]\right.$-palmitic, $\quad\left[9,10-{ }^{3} \mathrm{H}(\mathrm{N})\right]$-oleic $\quad$ and $\quad\left[5,6,8,9,11,12,14,15-{ }^{3} \mathrm{H}(\mathrm{N})\right]$ arachidonic acid, PerkinElmer, Waltham, MA) into TGs, PCs and CEs by thin layer chromatography were analysed as described in detail in Supplementary material.

Statistical analyses

The Kolmogorov-Smirnov test was used to test the normality of continuous variables. The independent two-sample Student's $t$ test and Mann-Whitney $U$ test were used to compare normally and non-normally distributed data, respectively. Normally distributed data were reported in means \pm standard error of means (SEM) while non-normally distributed data were reported as medians followed by interquartile ranges. Pearson's $\chi^{2}$ test was used to evaluate if the distribution of categorical variables differed between the groups. Pearson's correlation coefficients were calculated as a measure of statistical dependence between two variables after log-transformation if necessary. Statistical analyses were performed by using R 3.1.1 (http://www.r-project.org/), IBM SPSS Statistics 22.0.0.0 version (IBM, Armonk, NY), and GraphPad Prism 6.0f for Mac OS X (GraphPad Software, La Jolla, CA). A two-sided $p$ value of less than 0.05 indicated statistical significance.

The median and, after log2-transformation, the mean concentrations of all lipids were compared between groups based on TM6SF2 genotype. BenjaminiHochberg method was used for multiple hypotheses testing. ${ }^{29}$ Differences in concentrations of TGs were illustrated by a heatmap, where the color intensity of each cell represents the log2-transformed ratio of mean values of an individual $\mathrm{TG}$ in the TM6SF2 $2^{\mathrm{EK} / \mathrm{KK}}$ group divided by the mean values in the TM6SF2 ${ }^{\mathrm{EE}}$ group Fold-change of log2-transformed ratio of mean concentrations of lipids between the groups was visualized with respect to fatty acyl chain length and saturation. table.

\section{Results}

Characteristics of the study groups

Clinical characteristics of the TM6SF2 genotype groups are shown in Table 1 . The groups were similar with respect to age, gender and BMI but liver fat was 2-fold higher in the TM6SF2 ${ }^{\mathrm{EK} / \mathrm{KK}}$ than the TM6SF2 ${ }^{\mathrm{EE}}$ group (Table 1). Histologic liver fat percentage correlated with the sum of all liver TGs measured by UHPLC-MS $(\mathrm{r}=0.81, p<0.001)$

Fasting, 30 and $120 \mathrm{~min}$ glucose and insulin concentrations during the OGTT (Fig. 1) and the distribution of PNPLA3 rs738409 genotype (Table 1) were comparable between the TM6SF2 groups. The TM6SF2 $2^{\mathrm{EK} / \mathrm{KK}}$ tended to have higher prevalence of hepatic fibrosis compared to the $T M 6 S F 22^{\mathrm{EE}}$ group (60\% vs. $31 \%, p=0.09$ ).

\section{Hepatic TGs}

The increase in TGs in the TM6SF2 $2^{\mathrm{EK} / \mathrm{KK}}$ when compared to the $T_{M} 6 S 2^{\mathrm{EE}}$ group was due to an increase in TG species with 4854 carbons and $0-3$ double bonds, i.e. TGs containing predominantly saturated and monounsaturated long-chain fatty acids (Fig. 2). Concentrations of TGs with 58-60 carbons and 6-11 double bonds, i.e. TGs containing predominantly polyunsaturated fatty acids were decreased in the TM6SF2 $2^{\mathrm{EK} / \mathrm{KK}}$ when compared to the $T M 6 S F 2^{\mathrm{EE}}$ group (Fig. 2; Fig. S1; Table S1). Consequently, the fold-change of concentrations of TGs between the groups was significantly inversely correlated $(r=-0.27, p=0.0033)$ with

Table 1. Clinical characteristics of the study participants according to the TM6SF2 genotype at rs58542926.

\begin{tabular}{|c|c|c|}
\hline Total & TM6SF2 $^{\mathrm{EE}}(\mathrm{n}=80)$ & $T M 6 S F 2^{\mathrm{EK} / \mathrm{KK}}(\mathrm{n}=10)$ \\
\hline Age (years) & $45.2 \pm 0.9$ & $49.1 \pm 2.8$ \\
\hline Gender (\% women) & 71.3 & 70.0 \\
\hline BMI $\left(\mathrm{kg} / \mathrm{m}^{2}\right)$ & $45.4 \pm 0.7$ & $45.6 \pm 1.7$ \\
\hline Waist circumference $(\mathrm{cm})$ & $128.3(120.0-140.0)$ & $127.3(117.4-135.4)$ \\
\hline Liver fat (\%) & $13.8 \pm 2.0$ & $27.5 \pm 9.5^{*}$ \\
\hline Necroinflammatory grade $(0 / 1 / 2 / 3)$ & $67 / 13 / 0 / 0$ & $8 / 2 / 0 / 0$ \\
\hline Fibrosis stage $(0 / 1 / 2 / 3 / 4)$ & $55 / 23 / 2 / 0 / 0$ & $4 / 5 / 1 / 0 / 0$ \\
\hline Fibrosis stage $>0(\%)$ & 31 & $60(p=0.09)$ \\
\hline fS-insulin (mU/L) & $12.6(6.9-18.8)$ & $12.8(7.7-17.7)$ \\
\hline fP-glucose $(\mathrm{mmol} / \mathrm{L})$ & $5.6 \pm 0.1$ & $5.7 \pm 0.3$ \\
\hline $\mathrm{HbA}_{1 \mathrm{C}}(\%)$ & $5.7(5.5-6.0)$ & $5.7(5.5-6.1)$ \\
\hline $\mathrm{HbA}_{1 \mathrm{c}}(\mathrm{mmol} / \mathrm{mol})$ & $39(37-42)$ & $39(37-43)$ \\
\hline HOMA-IR ${ }^{\dagger}$ & $3.4 \pm 0.2$ & $3.6 \pm 0.7$ \\
\hline Matsuda index ${ }^{\dagger \dagger}$ & $55.4(36.0-104.9)$ & $56.9(42.2-74.3)$ \\
\hline fS-adiponectin $(\mu \mathrm{g} / \mathrm{ml})$ & $8.5 \pm 0.5$ & $10.1 \pm 1.3$ \\
\hline fP-total cholesterol (mmol/L) & $4.3 \pm 0.1$ & $4.2 \pm 0.2$ \\
\hline fP-triglycerides (mmol/L) & $1.24(0.91-1.69)$ & $1.34(0.97-1.60)$ \\
\hline fP-HDL cholesterol $(\mathrm{mmol} / \mathrm{L})$ & $1.13(0.96-1.37)$ & $1.09(0.96-1.55)$ \\
\hline fP-LDL cholesterol (mmol/L) & $2.6 \pm 0.1$ & $2.5 \pm 0.2$ \\
\hline P-AST (IU/L) & $30(25-37)$ & $34(30-41)$ \\
\hline P-ALT (IU/L) & $32(24-45)$ & $42(28-58)$ \\
\hline P-ALP (IU/L) & $64 \pm 2$ & $70 \pm 5$ \\
\hline P-GGT (U/L) & $28(21-45)$ & $50(26-66)$ \\
\hline P-albumin (g/L) & $38.2 \pm 0.3$ & $37.9 \pm 1.0$ \\
\hline B-platelets $\left(\right.$ x $\left.10^{9} / \mathrm{L}\right)$ & $252 \pm 6$ & $276 \pm 35$ \\
\hline PNPLA3 (n, CC/CG/GG) & $41 / 35 / 4$ & $6 / 3 / 1$ \\
\hline TM6SF2 (n, CC/CT/TT) & $80 / 0 / 0$ & $0 / 9 / 1^{* * *}$ \\
\hline
\end{tabular}

Data are in $n$, means \pm SEM or median (25th-75th percentile), and statistical tests are Student's $t$ test, Mann-Whitney $U$ test and Pearson's $\chi^{2}$-test, as appropriate. ${ }^{*} p \leqslant 0.05$. *** $p \leqslant 0.001$.

$\dagger$ Homeostasis model assessment of insulin resistance was calculated by formula: fS-Insulin x fP-Glucose/22.5 according to Matthews et al. ${ }^{25}$

${ }^{\dagger \dagger}$ Matsuda insulin sensitivity index was calculated from insulin and glucose concentrations measured at 0,30 and 120 min during the oral glucose tolerance test as proposed by DeFronzo and Matsuda. ${ }^{26}$ 


\section{JOURNAL OF HEPATOLOGY}

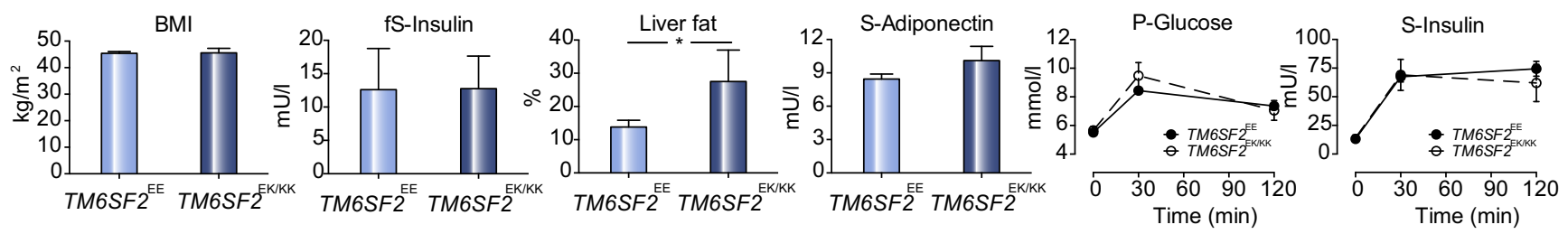

Fig. 1. Clinical characteristics of the groups based on the TM6SF2 genotype. BMI, fasting serum insulin, liver fat, serum adiponectin, plasma glucose concentrations during OGTT and serum insulin concentrations during OGTT. Light blue bars, black circles and solid lines, TM6SF2 ${ }^{\mathrm{EE}}(\mathrm{n}=80)$. Dark blue bars, open circles and broken lines, $T_{M 6 S F} 2^{\mathrm{EK} / \mathrm{KK}}(\mathrm{n}=10)$. Data are in means \pm SEM or median $\left(25\right.$ th-75th percentile); Student's $t$ test and Mann-Whitney $U$ test, as appropriate; ${ }^{*} p<0.05$.

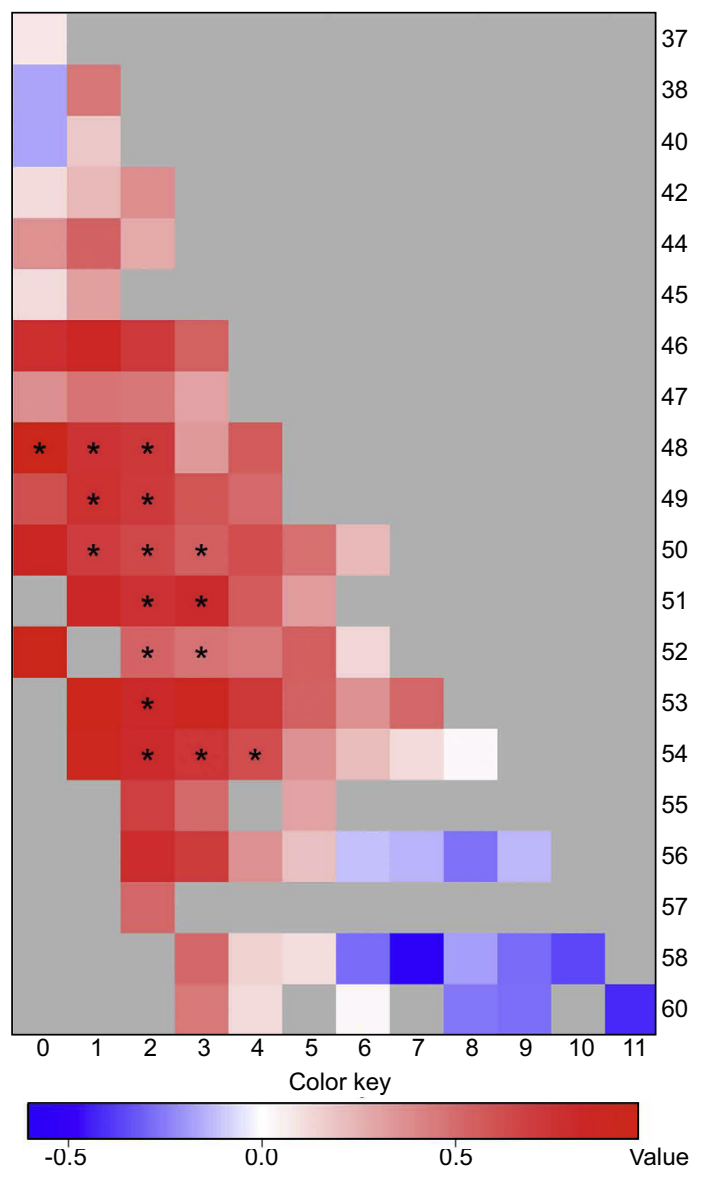

Fig. 2. Fold-change of hepatic triglycerides (TG) in the $\mathrm{TMGSF2}^{\mathrm{EK} / \mathrm{KK}}$ group compared to the TMGSF2 ${ }^{\mathrm{EE}}$ group. The color key shows the log of the ratio between means of the groups of an individual TG. The brighter the red color, the greater increase of absolute concentration of the individual TG in the TM6SF2 ${ }^{\mathrm{EK} / \mathrm{KK}}$ compared to the TM6SF2 ${ }^{\mathrm{EE}}$ group. The y-axis denotes the number of carbons and the $\mathrm{x}$-axis the number of double bonds. Student's $t$ test; ${ }^{*} p<0.05$.

the number of double bonds in TGs (Fig. S1). In vitro, TM6SF2 knockdown HuH-7 cells had 52\% lower TM6SF2 mRNA expression than control HuH-7 cells $(p<0.001)$ (Fig. $3 \mathrm{~A})$. Incorporation of palmitic acid (16:0, a saturated fatty acid) $(+117 \%, p<0.01)$ and oleic acid (18:1n-9, a monounsaturated fatty acid) $(+39 \%$, $p<0.05$ ) into TGs in the TM6SF2 knockdown HuH-7 cells were significantly increased, while that of arachidonic acid (20:4n-6, a polyunsaturated fatty acid) was significantly decreased $(-34 \%, p<0.001)$ (Fig. 3D). Transient silencing ( $-78 \%)$ of TM6SF2 in HuH-7 cells induced similar changes in fatty acid incorporation into TGs (Fig. S2A).
Hepatic CES

The concentration of total liver CEs was $20 \%$ higher in the TM6SF2 $2^{\mathrm{EK} / \mathrm{KK}}$ than the TM6SF2 ${ }^{\mathrm{EE}}$ group $(p<0.05)$ (Fig. 4). Among individual CE species, the concentration of $C E(16: 0)$ was significantly higher in the TM6SF2 ${ }^{\mathrm{EK} / \mathrm{KK}}$ group than the TM6SF2 $2^{\mathrm{EE}}$ group $(p<0.05)$, while those of $\mathrm{CE}(16: 1), \mathrm{CE}(18: 1)$ and $\mathrm{CE}(18: 2)$ tended to increase $(p=0.07, p=0.06$, and $p=0.05$, Fig. 4). In TM6SF2 knockdown HuH-7 cells, incorporation of palmitic acid (16:0) into CE was significantly $(+149 \%, p<0.05)$ and that of oleic acid $(18: 1)$ almost significantly $(+76 \%, p=0.06$, Fig. $3 \mathrm{E})$ increased. Transient silencing of TM6SF2 in HuH-7 cells induced similar changes in fatty acid incorporation into CEs (Fig. S2C).

\section{Hepatic PCs}

In the human liver samples, total PCs were significantly lower in the TM6SF2 $2^{\mathrm{EK} / \mathrm{KK}}$ than the TM6SF2 ${ }^{\mathrm{EE}}$ group $(-11 \%, p<0.05$; Fig. 4$)$. The fold-change of PCs between the groups was significantly inversely correlated with the number of double bonds $(\mathrm{r}=-0.40, p<0.001)$ but not with the number of carbons in PCs. Thus, polyunsaturated PCs were responsible for the decrease in total PCs (Fig. 4). To determine whether the decrease in polyunsaturated PCs was secondary to an increase in liver TG, we plotted liver TGs against individual polyunsaturated PC concentrations. As shown in Fig. S3, for any given liver TG concentration, those of polyunsaturated PCs were significantly lower in the TM6SF2 $2^{\mathrm{EK} / \mathrm{KK}}$ than the TM6SF2 ${ }^{\mathrm{EE}}$ group.

In TM6SF2 knockdown HuH-7 cells, the relative incorporation of arachidonic acid to PC was significantly lower than in control cells $(-32 \%, p<0.05$, Fig. $3 \mathrm{~F})$, while incorporations of palmitic $(+236 \%, p<0.001)$ and oleic $(+65 \%, p<0.05)$ acids were increased (Fig. 3D). Transient silencing of TM6SF2 expression in HuH-7 cells induced similar changes in fatty acid incorporation into PCs (Fig. S2B).

\section{Hepatic FFA}

The concentration of total hepatic FFA was significantly lower in the $T M 6 S F 2^{\mathrm{EK} / \mathrm{KK}}(700[595-822] \mathrm{nmol} / \mathrm{g})$ than the $T M 6 S F 2^{\mathrm{EE}}$ group $(904[755-1,098] \mathrm{nmol} / \mathrm{g}, p<0.01)$. Among the individual FFA, concentrations of palmitic (16:0, 286 [254-346] vs. 383 [307-456] nmol/g, $p<0.01)$, stearic $(18: 0,131$ [125-188] vs. 195 [160-231] nmol/g, $p<0.01)$, oleic $(18: 1,140$ [131-168] vs. 191 [141-247] nmol/g, $p<0.05)$ and linoleic $(18: 2,67$ [50-81] vs. 84 [67-102] nmol/g, $p<0.05$ ) acids were significantly lower in the TM6SF2 $2^{\mathrm{EK} / \mathrm{KK}}$ compared to the TM6SF2 $2^{\mathrm{EE}}$ group (Fig. 4). The relative proportion of arachidonic acid (20:4) in total FFA was significantly higher $(+15 \%, p<0.05)$ in the TM6SF2 ${ }^{\mathrm{EK} / \mathrm{KK}}$ compared to the TM6SF2 ${ }^{\mathrm{EE}}$ group (Fig. 4). 


\section{Research Article}
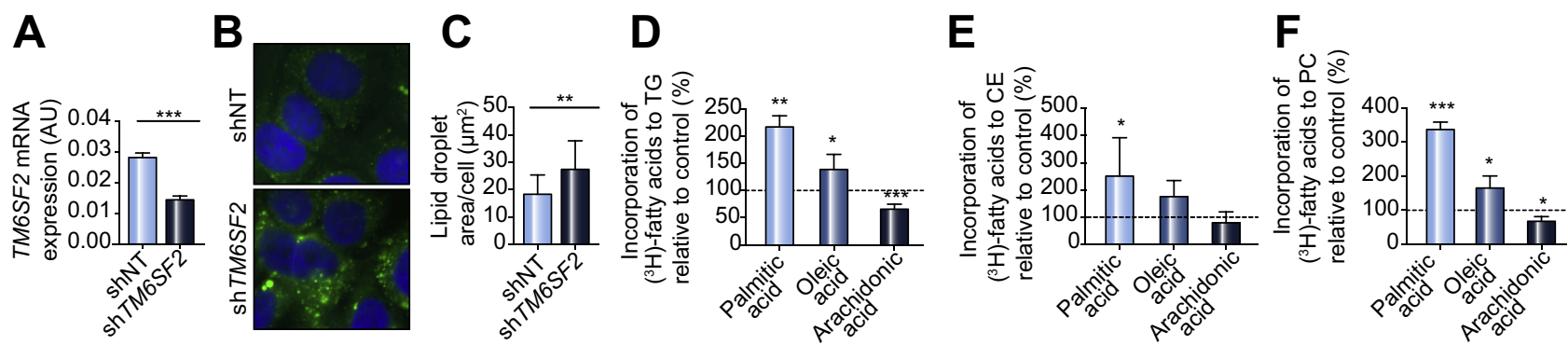

Fig. 3. In vitro experiments. (A) TM6SF2 mRNA expression in control shRNA (shNT) and TM6SF2 shRNA (shTM6SF2) expressing HuH-7 cells. The experiment was run twice in triplicates. (B) Staining of lipid droplets by BODIPY ${ }^{\circledR} 493 / 503$ in shNT and shTM6SF2 expressing HuH-7 cells and (C) the quantification of lipid droplet area per cell. The data represent the average of 150 cells from each set. The relative incorporation of $\left[{ }^{3} \mathrm{H}\right]$-palmitic acid, [ $\left.{ }^{3} \mathrm{H}\right]$-oleic acid and [ $\left.{ }^{3} \mathrm{H}\right]$-arachidonic acid into triglycerides (D), cholesteryl esters (E) and phosphatidylcholines (F) expressed in percentage relative to control $(\mathrm{n}=5)$. Data are shown as mean $\pm \mathrm{SD}$; Student's $t$ test; ${ }^{*} p<0.05$, ${ }^{* *} p<0.01$, $* * * p<0.001$.


Fig. 4. Differences in hepatic cholesteryl esters (CE), phosphatidylcholines (PC) and free fatty acids (FFA) between the groups. Concentrations of total and individual liver CEs (top left panel) and total liver PCs (top right panel) in TM6SF2 ${ }^{\mathrm{EK} / \mathrm{KK}}$ relative to TM6SF2 $2^{\mathrm{EE}}$ group. The height of the bars represent\% lipid concentration in $T M 6 S F 2^{\mathrm{EK} / \mathrm{KK}}$ relative to $T M 6 S F 2^{\mathrm{EE}}$ group. Linear regression between the number of double bonds (middle left panel) and carbons (middle right panel) in PCs and fold-change of liver PCs in the TM6SF $2^{\mathrm{EK} / \mathrm{KK}} v s$. the ${ }_{T M} 6 S F 2^{\mathrm{EE}}$ group (solid line). The intercepts and slopes denote deviation of this regression line from 0 . Each circle denotes a different lipid. Concentrations of different liver FFA (bottom left panel) and liver FFA as a percentage of total (bottom right panel) in $T M 6 S F 2^{\mathrm{EK} / \mathrm{KK}}$ and $T M 6 S F 2^{\mathrm{EE}}$. Data are in means $\pm \mathrm{SEM}$ or median (25th-75th percentile); Student's $t$ test and Mann-Whitney $U$ test, as appropriate; ${ }^{*} p<0.05,{ }^{* *} p<0.01$.
Serum TGs, PCs and CEs

In the serum, the fold-change of TGs in the $T M 6 S F 2^{\mathrm{EK} / \mathrm{KK}}$ compared to the TM6SF2 ${ }^{\mathrm{EE}}$ group tended to inversely correlate with the number of double bonds $(p=0.07)$ and number of carbons $(p=0.07)$ in TGs (Fig. 5). The intercepts of the fold-change regression lines between the groups were highly significantly, lower than 0 in serum TGs and PCs according to number of double bonds $(p<0.0001$ for both TGs and PCs) and carbons $(p<0.0001$ for both), but not in serum CEs (Fig. 5).

\section{Hepatic gene expression}

TM6SF2 mRNA expression was significantly downregulated in variant allele carriers (number of rs58542926 minor $(\mathrm{T})$ alleles vs. TM6SF2 expression, $\beta=-0.41 \pm 0.11, p<0.001$, Fig. S4). In pathway analyses, three pathways related to lipids, i.e. 'metabolism of lipids and lipoproteins', 'peroxisomal lipid metabolism' and 'triglyceride synthesis', were enriched with genes significantly correlated with TM6SF2 (Fig. 6; Table S3). Among individual genes which were positively co-expressed with TM6SF2 (and therefore decreased in TM6SF2 E167K carriers) were those associated with fatty acid (fatty acid synthase [FASN], acetyl-CoA carboxylase beta $[A C A C B]$ ) and triglyceride (diacylglycerol acyltransferase [DGAT]1, DGAT2) synthesis, and VLDL metabolism (apolipoprotein C-III [APOC3]), while those which were inversely co-expressed with TM6SF2 included genes related to fatty acid oxidation (carnitine palmitoyltransferase 1 [CPT1]), peroxisome proliferator-activator receptors alpha and gamma (PPARA, PPARG) and eicosanoid synthesis (arachidonate 5-lipoxygenase [ALOX5]) (Fig. 6).

\section{Discussion}

To the best of our knowledge, this is, the first study to compare human hepatic lipid profiles between carriers and non-carriers of the TM6SF2 E167K gene variant. Variant allele carriers had significantly higher hepatic TGs and CEs but lower total PCs due to a decrease in polyunsaturated PCs. Polyunsaturated fatty acids (PUFAs) were also deficient in serum and liver TGs but relatively enriched in liver FFA (Fig. 7). Consistent with this in vivo finding, using stably transduced and transiently silenced $\mathrm{HuH}-7$ cells as 


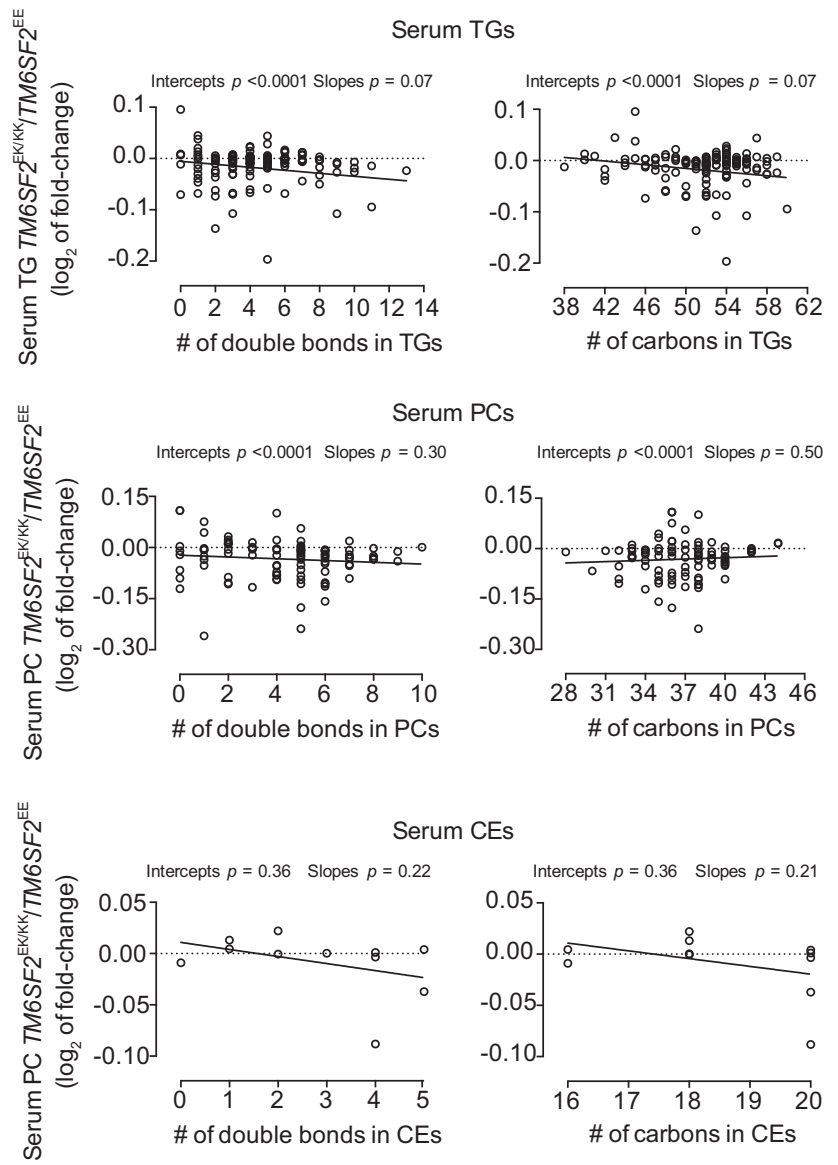

Fig. 5. Fold-change in serum triglycerides (TGs), phosphatidylcholines (PCs) and cholesteryl esters (CEs) in the TM6SF2 ${ }^{\mathrm{EK} / \mathrm{KK}}$ group compared to the TM6SF2 $^{\mathrm{EE}}$ group. Linear regression between the number of double bonds (left panels) and carbons (right panels) in serum TGs (top), PCs (middle), and CEs (bottom) and fold-change of respective serum lipids in the TM6SF2 $2^{\mathrm{EK} / \mathrm{KK}} v s$. the TM6SF2 ${ }^{\mathrm{EE}}$ group (solid line). The intercepts and slopes denote deviation of this regression line from 0 . Each circle denotes a different lipid.

in vitro hepatocyte models, TM6SF2 knockdown led to reduced incorporation of PUFAs into PCs and TGs. In the human liver, TM6SF2 was downregulated in variant allele carriers. Thus, downregulation of TM6SF2 increases liver TG and CE and decreases PC content by impairing lipid synthesis from PUFAs.

To examine the effect of the TM6SF2 E167K variant on human hepatic lipid content and composition, we divided the participants based on TM6SF2 genotype (TM6SF2 ${ }^{\mathrm{EK} / \mathrm{KK}} v$ s. TM6SF2 $\left.{ }^{\mathrm{EE}}\right)$. Carriers of this gene variant had 2-fold higher liver fat content but the groups did not differ with respect to HOMA-IR or insulin or glucose concentrations during the OGTT, or obesity. PNPLA3 genotype, which influences hepatic lipid composition, ${ }^{24,39}$ was also similar between the groups. Lack of features of insulin resistance between carriers and non-carriers of the TM6SF2 allele is similar to the finding of Kozlitina et al. in three independent cohorts. $^{2}$ In keeping with several previous studies involving $300,{ }^{5} 361^{6}$ and $691^{7}$ participants, there was no difference in fasting plasma triglyceride concentrations between the TM6SF2 genotype groups. However, in larger studies including $730^{9}$, $1,201^{12}$ and $4,587^{2}$ participants, the variant allele has been associated with decreased circulating triglycerides. In the present

\section{JOURNAL OF HEPATOLOGY}

Metabolism of lipids and lipoproteins

\author{
APOC3, ECHS1, SLC25A1, FASN, APOE, ACOX3, \\ DGAT1, DGAT2, APOA2, HADH, SLCO1B3, SLC25A17, \\ ECI1, ACOX2, LPIN3, NEU4, MED11, ARSA, \\ HSD11B1, GPD1, AKR1D1, BAAT, PHYH, ACADS, \\ GPAM, SLC27A5, AGPAT3, SCARB1, NPC1L1, CYP17A1, \\ CRAT, CYP8B1, MVK, ACACB, ABCC3, FDPS \\ CYP46A1, LSS, GPD2, GALC, FAR1, CYP7B1, \\ PPARA, LBR, ARNTL, ASAH1, SGPP1, CPT1A, \\ A2M, PPARG, OXCT1, GLIPR1, CLOCK, ALOX5 \\ LRP2, SPTLC2, AGPS
}

Triglyceride biosynthesis

SLC25A1, FASN, DGAT1, DGAT2, LPIN3, GPD1, GPAM, AGPAT3

\section{Peroxisomal lipid metabolism}

ACOX3, SLC25A17, ACOX2, PHYH, CRAT, FAR1, AGPS

Fig. 6. Hepatic lipid-related gene expression pathways enriched with genes co-expressing with TMGSF2. Names of the pathways are in bold and names of the genes in italics. Blue color denotes genes correlating positively (i.e. decreasing in TM6SF2 E167K carriers) and red color those correlating negatively with the expression of TM6SF2. Bold italics denote genes regulated by polyunsaturated fatty acids. ${ }^{41,42,52-55}$

study, variant allele carriers displayed deficiency of TGs and PCs in the circulation as compared to non-carriers in serum lipidomic analysis (Fig. 5). Deficiency of TGs and PCs in serum in the face of increased liver TG suggests defective hepatic lipid export, in line with previous data. ${ }^{2,8-10,12}$

In the liver of TM6SF2 E167K variant carriers compared to non-carriers (Fig. 2, Fig. 4; Fig. S1) and in vitro in hepatocytes (Fig. 3D, F), PUFAs in both PCs and TGs were decreased while the proportion of polyunsaturated arachidonic acid in total liver FFA was increased (Fig. 4). These results imply that the TM6SF2 E167K variant may regulate channeling of PUFAs into PC and TG synthesis. The first step in the incorporation of free PUFAs into these lipids is activation into a fatty acyl-CoA by an acyl-CoA synthetase (ACS) enzyme. ${ }^{33}$ The number of ACS enzymes has rapidly increased and includes at least 26 isoforms. ${ }^{34}$ These enzymes vary with respect to their substrate preference and subcellular localisation, and channel distinct fatty acids into different metabolic pathways. ${ }^{33,34}$ For example, knockdown of acyl-CoA synthetase long-chain family member 3 (ACSL3), which is expressed in the liver and intestine and localised in the endoplasmic reticulum similarly to TM6SF2, decreases incorporation of unsaturated fatty acids into PC, causes PC deficiency, and inhibits secretion of VLDL in HuH-7 cells. ${ }^{35}$

Regarding the mechanisms by which PC deficiency and excess polyunsaturated FFA interfere with VLDL metabolism, assembly, secretion and degradation of VLDL needs to be considered. The assembly of VLDL has two major steps. ${ }^{36}$ First, a nascent VLDL particle is formed in the ER, where it undergoes maturation to become a relatively lipid-poor small $\mathrm{VLDL}_{2}$ particle. ${ }^{36}$ This small particle is translocated to the Golgi complex and may be secreted as such, or undergoes a second lipidation step in which more TGs and CEs are added to the particle to form a highly-lipidated large $\mathrm{VLDL}_{1}$ particle. $^{36}$ 
Research Article
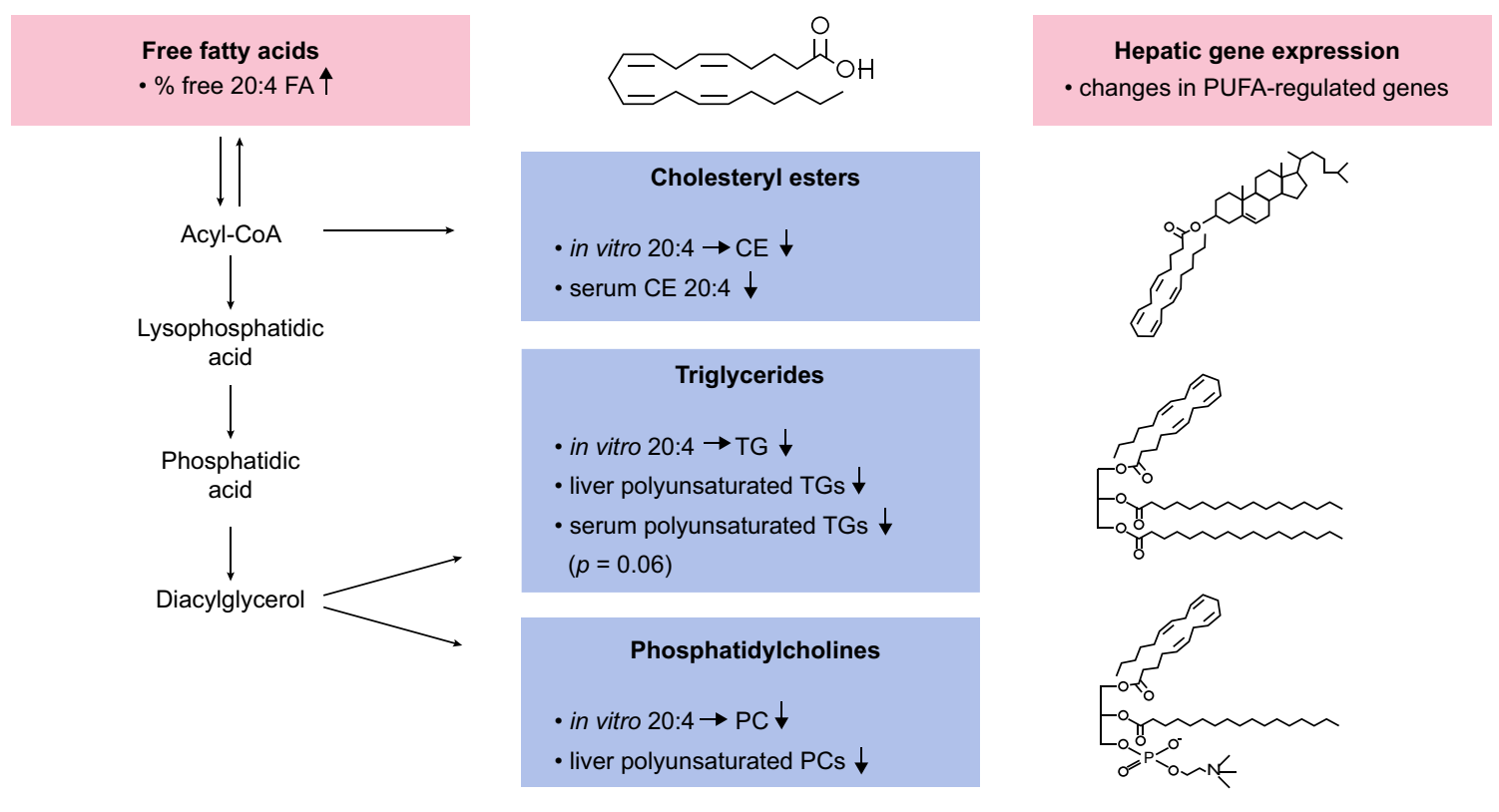

Fig. 7. Summary of changes in hepatic polyunsaturated fatty acid (PUFA) metabolism in vivo in TM6SF2 E167K gene variant carriers, and in vitro in TM6SF2 knockout HuH-7 cells. The percentage of hepatic free 20:4 fatty acid (FA) is increased in vivo. First step in synthesis of cholesteryl esters (CE), triglycerides (TG), and phosphatidylcholines (PC) is activation of free fatty acids into acyl-coenzyme A (CoA). These acyl-CoAs are esterified with cholesterol to form CEs. The in vitro incorporation of 20:4 into CEs is decreased, and concentrations of serum polyunsaturated CE are decreased in vivo. Acyl-CoAs are also utilized in de novo synthesis (via synthesis of lysophosphatidic acid, phosphatidic acid and diacylglycerol) of TGs and PCs. Incorporation of 20:4 into TGs and PCs is decreased in vitro, and hepatic concentrations of polyunsaturated TGs and PCs are decreased in vivo. Hepatic gene expression in vivo is characterized by changes in the expression of genes regulated by free PUFA. In summary, these in vivo and in vitro findings suggest that hepatic lipid synthesis from PUFA is impaired in TM6SF2 E167K gene variant carriers.

In TM6SF2 knockout mice, large VLDL particles are deficient in the circulation in the face of an unchanged or increased total number of particles secreted. ${ }^{37}$ This was suggested to reflect a defect during the second lipidation step. ${ }^{37}$ Consistent with these data in mice, in humans, large $\mathrm{VLDL}_{1}$ particles are markedly deficient in the circulation in TM6SF2 E167K variant allele carriers compared to non-carriers. ${ }^{11}$ This may be caused by PC deficiency $^{15}$ or excess polyunsaturated FFA. ${ }^{36,40}$ For example, in isolated mouse hepatocytes, reduced PC synthesis due to knockout of PEMT increases degradation and decreases secretion of particularly the highly-lipidated VLDL particles. ${ }^{38}$ Polyunsaturated FFA increase VLDL degradation by a similar mechanism. ${ }^{40}$ Thus, PC deficiency and the relative increase in polyunsaturated FFA in TM6SF2 variant carriers might lead to accumulation of TGs and CEs via degradation of highly-lipidated VLDL, in keeping with the conclusion of Hobbs and coworkers. ${ }^{37}$

Hepatic expression of TM6SF2 mRNA was decreased in E167K variant carriers (Fig. S4). Three lipid-related pathways were enriched with genes co-expressing with TM6SF2 (Fig. 6). Among individual genes, expression of those related to lipogenesis and lipid export (e.g. FASN, ACACB, DGAT2, DGAT1 and APOC3) was decreased, while expression of genes related to peroxisome proliferator-activated receptors, mitochondrial fatty acid oxidation, and eicosanoid synthesis (e.g. PPARA, PPARG, CPT1, ALOX5) was increased in the variant carriers (Fig. 6). Decreased expression of DGAT1 and DGAT2 in variant carriers is in line with previous in vitro data. ${ }^{3}$ Interestingly, virtually identical changes in these genes are induced by PUFAs, ${ }^{41}$ in part via transcriptional regulation by peroxisome proliferator-activated receptors ${ }^{42}$ (Fig. 6). These findings are consistent with the in vivo and in vitro data suggesting impaired hepatic incorporation of PUFA to TGs, CEs and PCs, increased relative concentrations of arachidonic acid in hepatic FFA, and deficiency of TGs and PCs in serum in TM6SF2 E167K gene variant carriers.

Although we observed PC deficiency both in the liver and in serum in humans carrying the TM6SF2 E167K variant compared to non-carriers, no changes in hepatic PC concentrations were observed in TM6SF2 knockout mice. ${ }^{37}$ This could be due to species difference. The main finding of the present study, i.e. impaired hepatic lipid synthesis from PUFAs in TM6SF2 E167K variant carriers, however, is entirely compatible with data in TM6SF2 knockout mice displaying reduced percentage of 20:4 and 22:6 in total hepatic lipids. ${ }^{37}$

Even though the sample size in the present study was large considering the difficulty in obtaining human liver samples, the number of variant allele carriers was low given the variant allele frequency of $7 \%$. The observed differences may thus underestimate true differences between carriers and non-carriers of the TM6SF2 E167K gene variant. We had no detailed dietary records of the patients. This is a limitation as PC is also an important dietary component. ${ }^{43}$ However, it is unlikely that genotype would affect intake of PC or its subsequent metabolism in gut lumen by bacteria to biologically active metabolites such as trimethylamine N-oxide. ${ }^{44}$

In keeping with human data of Liu et al., ${ }^{4}$ carriers of the TM6SF2 E167K variant tended to have increased liver fibrosis compared to non-carriers (60 vs. $31 \%, p=0.09$ ). PC deficiency and the relative excess of arachidonic acid found in the present study may contribute to NASH and fibrosis by several mechanisms. PC deficiency induced by a methionine-choline-deficient diet induces lipid peroxidation, stellate cell activation and fibrosis in rats. ${ }^{45}$ Excess arachidonic acid increases lipid peroxidation, 


\section{JOURNAL OF HEPATOLOGY}

and apoptosis, ${ }^{46}$ and stellate cell collagen expression in vitro, ${ }^{47}$ while arachidonic acid deficiency has opposite effects. ${ }^{48}$ Arachidonic acid can be metabolized into proinflammatory eicosanoids, ${ }^{33}$ which circulating concentrations are increased in participants with $\mathrm{NASH}^{49,50}$ Increased liver fat content per se may increase the risk of NASH, as evidenced by recent prospective liver biopsy data. ${ }^{51}$

In conclusion, hepatic synthesis of polyunsaturated fatty acidcontaining lipids is impaired in TM6SF2 E167K gene variant carriers and leads to deficiency of polyunsaturated PCs and excess polyunsaturated FFA in the human liver. These changes, based on abundant experimental data, could impair VLDL lipidation via increased degradation, and explain the phenotype characterizing TM6SF2 E167K carriers i.e. hepatic steatosis, lack of circulating hypertriglyceridemia and an increased risk of NASH.

\section{Financial support}

This study was supported by research grants from the Faculty of Medicine of the University of Helsinki and the Helsinki University Hospital (PL), the Academy of Finland (HY, LG, TT), EU/EFPIA Innovative Medicines Initiative Joint Undertaking (EMIF grant no. 115372, HY), EU H2020 EPoS 634413 (HY, QMA), the Sigrid Juselius (HY, LG), EVO (HY), Liv och Hälsa (VO), the Magnus Ehrnrooth (PANH), the Finnish Cardiovascular Research (VO), and the Cardiff University SIURI Seedcorn fund (YZ) Foundations

\section{Conflict of interest}

The authors who have taken part in this study declared that they do not have anything to disclose regarding funding or conflict of interest with respect to this manuscript.

Please refer to the accompanying ICMJE disclosure forms for further details.

\section{Authors' contributions}

PL - study concept and design; acquisition of data; analysis and interpretation of data; drafting of the manuscript; critical revision of the manuscript for important intellectual content; statistical analysis. YZ, OPD, TT, PV - bioinformatics and statistical analyses; interpretation of data; critical revision of the manuscript for important intellectual content. PANH, VO - in vitro experiments; interpretation of data; critical revision of the manuscript for important intellectual content. TH, AA, AJ, ML, LA, JA, MOM, MO, LG - acquisition of data; critical revision of the manuscript for important intellectual content. ES, JMP, QMA - obtained funding; critical revision of the manuscript for important intellectual content. HY - study concept and design; analysis and interpretation of data; drafting of the manuscript; critical revision of the manuscript for important intellectual content; obtained funding; study supervision.

\section{Acknowledgements}

We thank Anne Salo, Aila Karioja-Kallio, Mia Urjansson, Katja Sohlo, Erja Juvonen, Anna-Liisa Ruskeepää, Ulla Lahtinen, Heli Nygren, Ismo Mattila and Anette Unterman for their excellent technical assistance.

\section{Supplementary data}

Supplementary data associated with this article can be found, in the online version, at http://dx.doi.org/10.1016/j.jhep.2017.02. 014 .

\section{References}

Author names in bold designate shared co-first authorship

[1] Yki-Järvinen H. Non-alcoholic fatty liver disease as a cause and a consequence of metabolic syndrome. Lancet Diabetes Endocrinol 2014:2:901-910.

[2] Kozlitina J, Smagris E, Stender S, Nordestgaard BG, Zhou HH, TybjærgHansen A, et al. Exome-wide association study identifies a TM6SF2 variant that confers susceptibility to nonalcoholic fatty liver disease. Nat Genet 2014;46:352-356.

[3] Mahdessian H, Taxiarchis A, Popov S, Silveira A, Franco-Cereceda A, Hamsten $A$, et al. TM6SF2 is a regulator of liver fat metabolism influencing triglyceride secretion and hepatic lipid droplet content. Proc Natl Acad Sci USA 2014;111:8913-8918.

[4] Liu YL, Reeves HL, Burt AD, Tiniakos D, McPherson S, Leathart JB, et al TM6SF2 rs58542926 influences hepatic fibrosis progression in patients with non-alcoholic fatty liver disease. Nat Commun 2014;5:4309.

[5] Zhou Y, Llauradó G, Orešič M, Hyötyläinen T, Orho-Melander M, YkiJärvinen $\mathrm{H}$. Circulating triacylglycerol signatures and insulin sensitivity in NAFLD associated with the E167K variant in TM6SF2. J Hepatol 2015;62:657-663.

[6] Sookoian S, Castaño GO, Scian R, Mallardi P, Fernández Gianotti T, Burgueño AL, et al. Genetic variation in transmembrane 6 superfamily member 2 and the risk of nonalcoholic fatty liver disease and histological disease severity. Hepatology 2015;61:515-525.

[7] Goffredo M, Caprio S, Feldstein AE, D’Adamo E, Shaw MM, Pierpont B, et al Role of TM6SF2 rs58542926 in the pathogenesis of nonalcoholic pediatric fatty liver disease: A multiethnic study. Hepatology 2016;63:117-125.

[8] Grandone A, Cozzolino D, Marzuillo P, Cirillo G, Di Sessa A, Ruggiero L, et al TM6SF2 Glu167Lys polymorphism is associated with low levels of LDLcholesterol and increased liver injury in obese children. Pediatr Obes 2016:11:115-119.

[9] Eslam M, Mangia A, Berg T, Chan HL, Irving WL, Dore GJ, et al. Diverse impacts of the rs58542926 E167K variant in TM6SF2 on viral and metabolic liver disease phenotypes. Hepatology 2016. http://dx.doi.org/10.1002/ hep. 28475.

[10] Mancina RM, Sentinelli F, Incani M, Bertoccini L, Russo C, Romeo S, et al. Transmembrane-6 superfamily member 2 (TM6SF2) E167K variant increases susceptibility to hepatic steatosis in obese children. Dig Liver Dis 2016;48:100-101.

[11] Musso G, Cassader M, Paschetta E, Gambino R. TM6SF2 may drive postprandial lipoprotein cholesterol toxicity away from the vessel walls to the liver in NAFLD. J Hepatol 2016;64:979-981.

[12] Dongiovanni P, Petta S, Maglio C, Fracanzani AL, Pipitone R, Mozzi E, et al TM6SF2 gene variant disentangles nonalcoholic steatohepatitis from cardiovascular disease. Hepatology 2015;61:506-514.

[13] Holmen OL, Zhang H, Fan Y, Hovelson DH, Schmidt EM, Zhou W, et al Systematic evaluation of coding variation identifies a candidate causal variant in TM6SF2 influencing total cholesterol and myocardial infarction risk. Nat Genet 2014;46:345-351.

[14] Sun Z, Lazar MA. Dissociating fatty liver and diabetes. Trends Endocrinol Metab 2013;24:4-12.

[15] Cole LK, Vance JE, Vance DE. Phosphatidylcholine biosynthesis and lipoprotein metabolism. Biochim Biophys Acta 2012;1821:754-761.

[16] Rong X, Wang B, Dunham MM, Hedde PN, Wong JS, Gratton E, et al. Lpcat3dependent production of arachidonoyl phospholipids is a key determinant of triglyceride secretion. Elife 2015;25:4.

[17] Sha W, da Costa KA, Fischer LM, Milburn MV, Lawton KA, Berger A, et al Metabolomic profiling can predict which humans will develop liver dysfunction when deprived of dietary choline. FASEB J 2010;24:2962-2975.

[18] Rinella ME, Green RM. The methionine-choline deficient dietary model of steatohepatitis does not exhibit insulin resistance. J Hepatol 2004;40:47-51.

[19] Song J, da Costa KA, Fischer LM, Kohlmeier M, Kwock L, Wang S, et al. Polymorphism of the PEMT gene and susceptibility to nonalcoholic fatty liver disease (NAFLD). FASEB J 2005;19:1266-1271. 


\section{Research Article}

[20] Romeo S, Cohen JC, Hobbs HH. No association between polymorphism in PEMT (V175M) and hepatic triglyceride content in the Dallas Heart Study. FASEB J 2006;20:2180.

[21] Zeisel SH. People with fatty liver are more likely to have the PEMT rs7946 SNP, yet populations with the mutant allele do not have fatty liver. FASEB J 2006;20:2181-2182.

[22] Dong H, Wang J, Li C, Hirose A, Nozaki Y, Takahashi M, et al. The phosphatidylethanolamine N-methyltransferase gene V175M single nucleotide polymorphism confers the susceptibility to NASH in Japanese population. J Hepatol 2007;46:915-920.

[23] Spencer MD, Hamp TJ, Reid RW, Fischer LM, Zeisel SH, Fodor AA. Association between composition of the human gastrointestinal microbiome and development of fatty liver with choline deficiency. Gastroenterology 2011;140:976-986.

[24] Luukkonen PK, Zhou Y, Sädevirta S, Leivonen M, Arola J, Orešič M, et al. Hepatic ceramides dissociate steatosis and insulin resistance in patients with non-alcoholic fatty liver disease. J Hepatol 2016;64:1167-1175.

[25] Alberti KG, Zimmet PZ. Definition, diagnosis and classification of diabetes mellitus and its complications. Part 1: diagnosis and classification of diabetes mellitus provisional report of a WHO consultation. Diabet Med 1998; 15:539-553.

[26] Matthews DR, Hosker JP, Rudenski AS, Naylor BA, Treacher DF, Turner RC Homeostasis model assessment: insulin resistance and beta-cell function from fasting plasma glucose and insulin concentrations in man. Diabetologia 1985;28:412-419.

[27] DeFronzo RA, Matsuda M. Reduced time points to calculate the composite index. Diabetes Care 2010;33:e93.

[28] Brunt EM, Janney CG, Di Bisceglie AM, Neuschwander-Tetri BA, Bacon BR. Nonalcoholic steatohepatitis: a proposal for grading and staging the histological lesions. Am J Gastroenterol 1999;94:2467-2474.

[29] Hochberg Y, Benjamini Y. More powerful procedures for multiple significance testing. Stat Med 1990;9:811-818.

[33] Cooper DE, Young PA, Klett EL, Coleman RA. Physiological Consequences of Compartmentalized Acyl-CoA Metabolism. J Biol Chem 2015;290:20023-20031.

[34] Pérez-Chacón G, Astudillo AM, Balgoma D, Balboa MA, Balsinde J. Control of free arachidonic acid levels by phospholipases A2 and lysophospholipid acyltransferases. Biochim Biophys Acta 2009;1791: 1103-1113.

[35] Yao H, Ye J. Long chain acyl-CoA synthetase 3-mediated phosphatidylcholine synthesis is required for assembly of very low density lipoproteins in human hepatoma Huh7 cells. J Biol Chem 2008;283:849-854.

[36] Olofsson SO, Borén J. Apolipoprotein B secretory regulation by degradation. Arterioscler Thromb Vasc Biol 2012;32:1334-1338.

[37] Smagris E, Gilyard S, BasuRay S, Cohen JC, Hobbs HH. Inactivation of Tm6sf2, a gene defective in fatty liver disease, impairs lipidation but not secretion of very low density lipoproteins. J Biol Chem 2016;291:10659-10676.

[38] Noga AA, Zhao Y, Vance DE. An unexpected requirement for phosphatidylethanolamine $\mathrm{N}$-methyltransferase in the secretion of very low density lipoproteins. J Biol Chem 2002;277:42358-42365.
[39] Peter A, Kovarova M, Nadalin S, Cermak T, Königsrainer A, Machicao F, et al. PNPLA3 variant I148M is associated with altered hepatic lipid composition in humans. Diabetologia 2014:57:2103-2107.

[40] Fisher EA, Pan M, Chen X, Wu X, Wang H, Jamil H, et al. The triple threat to nascent apolipoprotein B. Evidence for multiple, distinct degradative pathways. J Biol Chem 2001;276:27855-27863.

[41] Sampath H, Ntambi JM. Polyunsaturated fatty acid regulation of genes of lipid metabolism. Annu Rev Nutr 2005;25:317-340.

[42] Forman BM, Chen J, Evans RM. Hypolipidemic drugs, polyunsaturated fatty acids, and eicosanoids are ligands for peroxisome proliferator-activated receptors alpha and delta. Proc Natl Acad Sci USA 1997;94:4312-4317.

[43] Cohn JS, Wat E, Kamili A, Tandy S. Dietary phospholipids, hepatic lipid metabolism and cardiovascular disease. Curr Opin Lipidol 2008;19:257-262.

[44] Wang Z, Klipfell E, Bennett BJ, Koeth R, Levison BS, Dugar B, et al. Gut flora metabolism of phosphatidylcholine promotes cardiovascular disease. Nature 2011;472:57-63.

[45] George J, Pera N, Phung N, Leclercq I, Yun Hou J, Farrell G. Lipid peroxidation, stellate cell activation and hepatic fibrogenesis in a rat model of chronic steatohepatitis. J Hepatol 2003;39:756-764.

[46] Chen O, Galleano M, Cederbaum AI. Cytotoxicity and apoptosis produced by arachidonic acid in Hep G2 cells overexpressing human cytochrome P4502E1. J Biol Chem 1997;272:14532-14541.

[47] Nieto N, Greenwel P, Friedman SL, Zhang F, Dannenberg AJ, Cederbaum AI. Ethanol and arachidonic acid increase alpha 2(I) collagen expression in rat hepatic stellate cells overexpressing cytochrome P450 2E1. Role of $\mathrm{H}_{2} \mathrm{O}_{2}$ and cyclooxygenase-2. J Biol Chem 2000;275:20136-20145.

[48] Ishihara K, Miyazaki A, Nabe T, Fushimi H, Iriyama N, Kanai S, et al. Group IVA phospholipase A2 participates in the progression of hepatic fibrosis. FASEB J 2012;26:4111-4121.

[49] Loomba R, Quehenberger O, Armando A, Dennis EA. Polyunsaturated fatty acid metabolites as novel lipidomic biomarkers for noninvasive diagnosis of nonalcoholic steatohepatitis. J Lipid Res 2015;56:185-192.

[50] Puri P, Wiest MM, Cheung O, Mirshahi F, Sargeant C, Min HK, et al. The plasma lipidomic signature of nonalcoholic steatohepatitis. Hepatology 2009;50:1827-1838.

[51] Singh S, Allen AM, Wang Z, Prokop LJ, Murad MH, Loomba R. Fibrosis progression in nonalcoholic fatty liver $v$ s. nonalcoholic steatohepatitis: a systematic review and meta-analysis of paired-biopsy studies. Clin Gastroenterol Hepatol 2015;13:643-654 e1-e9.

[52] Kabir Y, Ide T. Activity of hepatic fatty acid oxidation enzymes in rats fed alpha-linolenic acid. Biochim Biophys Acta 1996;1304:105-119.

[53] Iacobazzi V, Infantino V, Palmieri F. Transcriptional regulation of the mitochondrial citrate and carnitine/acylcarnitine transporters: two genes involved in fatty acid biosynthesis and $\beta$-oxidation. Biology (Basel) 2013;2:284-303.

[54] Mathur SN, Watt KR, Field FJ. Regulation of intestinal NPC1L1 expression by dietary fish oil and docosahexaenoic acid. J Lipid Res 2007;48:395-404.

[55] Rådmark O, Werz O, Steinhilber D, Samuelsson B. 5-Lipoxygenase, a key enzyme for leukotriene biosynthesis in health and disease. Biochim Biophys Acta 2015;1851:331-339. 